\title{
TSO-DSO Operational Planning Coordination through Surrogate Lagrangian Relaxation
}

\author{
Mikhail A. Bragin, Member, IEEE, and Yury Dvorkin, Member, IEEE
}

\begin{abstract}
With the proliferation of distributed energy resources (DERs), located at the Distribution System Operator (DSO) level, uncertainties propagate from Transmission System Operator (TSO) to DSOs and vice versa. Therefore, to enable interoperability, while ensuring higher flexibility and costefficiency, both systems need to be efficiently coordinated to operate in sync. Moreover, because of the intermittency of renewable generation, voltages exhibit fluctuations thus necessitating the inclusion of AC power flow. Difficulties behind creating such TSO-DSO coordination include the combinatorial nature of the operational planning problem involved at the transmission level as well as the nonlinearity of $\mathrm{AC}$ power flow within both systems. These considerations significantly increase the complexity even under the deterministic setting. In this paper, a deterministic TSO-DSO operational planning coordination problem is considered and a novel decomposition and coordination approach is developed. Within the new method, the problem is decomposed into TSO and DSO subproblems, which are efficiently coordinated by updating Lagrangian multipliers. The nonlinearities at the TSO level caused by AC power flow constraints are resolved through a novel dynamic linearization. Numerical results based on the coordination of 118-bus TSO system with 32 DSO 34-bus systems indicate that both systems benefit from the coordination.
\end{abstract}

Index Terms-Distribution System Operations, Transmission System Operations, Surrogate Lagrangian Relaxation, Surrogate Absolute-Value Lagrangian Relaxation, TSO-DSO Coordination.

\section{INTRODUCTION}

$\mathbf{T}$ HE widespread and rapid proliferation of distributed energy resources (DERs) such as wind and solar, which are typically managed by Distribution System Operators (DSOs), has a far-reaching impact on the traditional power system paradigms and broad implications on the entire power grid [1]-[3]. This proliferation is fuelled by the emerging IoT technologies enabling the integration of DERs into the grid [3] and the distributed generation is expected to provide services to the entire grid [4]-[6]. Through the coordination of TSO and DSOs, potential flexibility benefits enabled by DERs [1], [3]-[11] can be achieved, for example, by adjusting DERs' operation point, voltage fluctuation and congestion issues can be mitigated [9]. Moreover, cost-benefits can be exploited [12]-[14].

However, while transmission renewable resources such as bulk wind and solar generation, DSOs' distributed energy resources (DERs) are to a large extent not observable by

M. A. Bragin is with the Department of Electrical and Computer Engineering, University of Connecticut, Storrs, CT, 06269, USA; e-mail: mikhail.bragin@uconn.edu.

Y. Dvorkin is with the Department of Electrical and Computer Engineering at New York University's Tandon School of Engineering, Brooklyn, NY, 11201, USA; e-mail: dvorkin@nyu.edu. the TSO. However, as mentioned above, since the DSOs are expected to actively engage in TSO operations [15], [16], uncertainties propagate from distribution to transmission level and vice versa. Therefore, both systems need to be operated in sync. With high penetration levels of renewables, the interoperability of TSO and DSOs becomes challenging and an appropriate coordination mechanism for coordinating TSO and DSOs is needed. Furthermore, the intermittent nature of renewables has led the voltage and frequency fluctuations and affected the nature of interactions among distribution (DSO) and transmission (TSO) systems. To control voltage, the consideration of AC power flow is necessary within both systems. These considerations significantly increase TSO-DSO coordination problem complexity even under the deterministic setting, and current methods may not be able to ensure efficient TSO-DSO coordination while considering unit commitment decisions and $\mathrm{AC}$ power flow constraints.

The TSO-DSO operational planning coordination is thus challenging in view of the following difficulties: 1 ) the presence of multiple DSOs that need to be coordinated together with the TSO; 2) the consideration of binary UC decision variables at the TSO level, which lead to the drastic increase of complexity; 3) the consideration of nonlinear AC power flows at the TSO level, which contributes to the non-convexity of the problem. While the presence of AC power flow at the DSO can be handled by using the exact second-order cone (SOC) relaxation method [17], [18], the method is valid only for radial topologies and cannot be used to handle the AC power flow at the TSO level with the meshed topology of the transmission network. Most research papers to date do not consider binary commitment decision variables [1], [19]-[29]. A review of the above TSO-DSO coordination models and methods can be found at [30]. There have been a few attempts at coordinating TSO and several DSOs while considering binary unit commitment variables [3] and [31]-[33]. However, within [1], [3], [23]-[29], [31]-[33], DC power flow was considered at the transmission level.

In Section II, the novel TSO-DSO operational planning coordination problem is formulated while considering $\mathrm{AC}$ power flow within each system (specifically, exact AC power flow constraints are incorporated within the unit commitment problem solved at the TSO level). Within TSO, generation capacity and ramp-rate, as well as AC power flow constraints (in rectangular coordinates), are considered. Within DSOs, generation capacity constraints, second-order semi-definite relaxation for AC power flow [17], [18], and nodal AC power flow balance constraints are considered. To model the coupling between TSO and DSOs, interface power exchange constraints 
are considered.

In Section III, the solution methodology is developed to efficiently coordinate TSO and DSOs while resolving nonlinearity and non-convexity difficulties introduced by $\mathrm{AC}$ power flow building upon the Surrogate Lagrangian Relaxation (SLR) decomposition and coordination approach [34]. The method overcame all difficulties of previous coordination LR-based methods for solving mixed-integer programming (MIP) problems, without requiring to solve all subproblems to update multipliers and guaranteeing smooth convergence. ${ }^{1}$ The efficient coordination of TSO and DSOs within the new method rests upon the following fundamental principles: 1) exponential reduction of complexity upon decomposition, 2) contraction mapping that ensures convergence of Lagrangian multipliers and 3) dynamic linearization of $\mathrm{AC}$ power flow constraints with proximal-like terms to ensure feasibility. These ideas are operationalized by relaxing nodal flow balance constraints that couple nodes within TSO, and by relaxing interface power exchange constraints that couple TSO and DSOs to decompose the problem in TSO and DSO subproblems. The resulting subproblems are solved independently of each other with much-reduced complexity and are coordinated by updating Lagrangian multipliers. Convergence is accelerated through the use of "absolute-value" penalties (following [36]) which are exactly linearized. After the linearization of penalties, the relaxed problem is still nonlinear because of the presence of nonlinear AC power flow constraints. Active and reactive power flow constraints formulated in rectangular coordinates $[38]-[42]$ are dynamically linearized in a novel way by alternatively fixing voltages at adjacent nodes while ensuring convergence and feasibility through a proximal-like technique with an $l_{1}$-norm which preserves the overall linearity of the problem and is amenable for the use of MILP solvers.

In Numerical Testing Section IV, a series of cases are considered to illustrate several important features of the new method. In Example 1, a small 4-bus system with 2 generators and 1 DSO is considered to demonstrate the convergence of multipliers. In Example 2, a small 9-bus system is considered [43] to demonstrate the efficiency of linearization that enables the satisfaction of nonlinear constraints by using MILP methods. In Example 3, to test scalability, the topology of the IEEE 118-bus system [43] and 32 DSOs based on the topology of the standard IEEE 34-bus system [43] is considered. Numerical results demonstrate that with such TSO-DSO coordination, both systems benefit and the coordination is efficient.

\section{TSO-DSO OPTIMIZATION MODEL}

In subsection A, the TSO problem formulation is presented. In subsection $\mathrm{B}$, the DSO problem formulations is presented. In subsection $\mathrm{C}$, the overall TSO-DSO coordination problem is developed.

\footnotetext{
${ }^{1}$ The method has been further improved and has been used to successfully solve large-scale unit commitment problems with DC power flow [35] as well as other mixed-integer programming (MIP) problems such as assignment and scheduling [36] and DNN training [37] while showing an advantage over such methods as branch-and-cut [35] and ADMM [36]- [37].
}

\section{A. TSO Model}

Consider a transmission network with meshed topology operated by a transmission system operator (TSO) with a set of interconnections with several distribution networks (DSOs). Let $\mathcal{T}$ be the lookahead horizon, e.g., $\mathcal{T}=1,2, \ldots, T$. Within the TSO, let $\mathcal{B}^{T}$ be a set of buses indexed by $b^{T}, \mathcal{I}_{b^{T}}^{T}$ be a set of generators at bus $b^{T}$ indexed by $i, \mathcal{J}^{T}\left(\mathcal{J}^{T} \subset \mathcal{B}^{T}\right)$ be a set of interconnections/root buses, whereby distribution networks are connected to the transmission network, indexed by $j, \mathcal{L}^{T}$ be a sets of transmission lines indexed by $l$.

Objective. The TSO aims to minimize the total generation cost as well as the total cost of power exchange at the interface of the TSO and DSOs:

$$
\begin{aligned}
& \min _{\mathbf{G}^{T}, \mathbf{P}^{T}, \mathbf{Q}^{T}}\left\{O^{T}\left(\mathbf{G}^{T}, \mathbf{P}^{T}, \mathbf{Q}^{T}\right)\right\}= \\
& \min _{\mathbf{G}^{T}, \mathbf{P}^{T}, \mathbf{Q}^{T}}\left\{\sum_{t \in \mathcal{T}, b^{T} \in \mathcal{B}^{T}, i \in \mathcal{I}_{b T}^{T}}\left(C_{i, t}^{T, p} g_{i, t}^{T, p}+C_{i, t}^{T, q} g_{i, t}^{T, q}\right)+\right. \\
&\left.\sum_{t \in \mathcal{T}, j \in \mathcal{J}^{T}}\left(C_{j, t}^{p} p_{j, t}^{T}+C_{j, t}^{q} q_{j, t}^{T}\right)\right\}
\end{aligned}
$$

where $\mathbf{G}^{T}=\left\{g_{i, t}^{T, p}, g_{i, t}^{T, q}\right\}$ is a vector of active and reactive generation levels with the corresponding generation costs $C_{i, t}^{T, p}$ and $C_{i, t}^{T, q}, \mathbf{P}^{T}=\left\{p_{j, t}^{T}\right\}$ is a vector of active interface power exchange amounts with the corresponding DSO $j^{\text {th }}$ bids $C_{j, t}^{p}$ and $\mathbf{Q}^{T}=\left\{q_{j, t}^{T}\right\}$ is a vector of reactive interface power exchange amount with the corresponding DSO $j^{\text {th }}$ bids $C_{j, t}^{q}$. Interface power exchange amounts $p_{j, t}^{T}$ and $q_{j, t}^{T}$ are positive if power exchange flow is from DSO to TSO, i.e., TSO buys power, and negative, otherwise.

The optimization (1) is subject to the following constraints: Generation Capacity Constraints. Active $g_{i, t}^{T, p}$ and reactive $g_{i, t}^{T, q}$ generation levels of unit $i$ are constrained as:

$$
\begin{aligned}
& \underline{G}_{i}^{T, p} \cdot x_{i, t} \leq g_{i, t}^{T, p} \leq \bar{G}_{i}^{T, p} \cdot x_{i, t}, \\
& \underline{G}_{i}^{T, q} \cdot x_{i, t} \leq g_{i, t}^{T, q} \leq \bar{G}_{i}^{T, q} \cdot x_{i, t},
\end{aligned}
$$

where where $x_{i, t} \in\{0,1\}$ are binary commitment decision variables and the minimum and maximum active power limits are $\underline{G}_{i}^{T, p}$ and $\bar{G}_{i}^{T, p}$, while the minimum and maximum reactive power limits are $\underline{G}_{i}^{T, q}$ and $\bar{G}_{i}^{T, q}$

Ramp-Rate Constraints. Ramp-rate constraints require that the change of power generation levels between two consecutive time periods does not exceed ramp rates $R_{i}^{p / q}$ :

$$
\begin{gathered}
g_{i, t}^{T, p}-g_{i, t-1}^{T, p} \leq R_{i}^{p} \cdot x_{i, t-1}+\left(\underline{G}_{i}^{T, p}+\frac{R_{i}^{p}}{2}\right) \cdot\left(x_{i, t}-x_{i, t-1}\right), \\
g_{i, t}^{T, q}-g_{i, t-1}^{T, q} \leq R_{i}^{q} \cdot x_{i, t-1}+\left(\underline{G}_{i}^{T, q}+\frac{R_{i}^{q}}{2}\right) \cdot\left(x_{i, t}-x_{i, t-1}\right), \\
g_{i, t-1}^{T, p}-g_{i, t}^{T, p} \leq R_{i}^{p} \cdot x_{i, t}+\left(\underline{G}_{i}^{T, p}+\frac{R_{i}^{p}}{2}\right) \cdot\left(x_{i, t-1}-x_{i, t}\right), \\
g_{i, t-1}^{T, q}-g_{i, t}^{T, q} \leq R_{i}^{q} \cdot x_{i, t}+\left(\underline{G}_{i}^{T, q}+\frac{R_{i}^{q}}{2}\right) \cdot\left(x_{i, t-1}-x_{i, t}\right) .
\end{gathered}
$$

For a complete set of tightened ramp-rate constraints, refer to [44]. 
Nodal Power Flow Balance Constraints. For every node $b^{T}$, the net active/reactive power generated and transmitted to the node should be equal to the net power consumed and transmitted from the node $b^{T}$ :

$$
\sum_{\substack{i \in \mathcal{I}_{b^{T}}^{T} \\ g_{i, t}}}^{T, p}+\sum_{\substack{l=1: \\ r(l)=b^{T}}}^{L} f_{l, t}^{T, p}+p_{b^{T}, t}^{T}=L_{b^{T}, t}^{T, p}+\sum_{\substack{l=1: \\ s(l)=b^{T}}}^{L} f_{l, t}^{T, p} .
$$

If bus $b^{T}$ does not contain generators, then $\sum_{i \in \mathcal{I}_{b T}^{T}} g_{i, t}^{T, p}=0$ if bus $b^{T}$ is not a root bus $\left(b^{T} \notin \mathcal{J}^{T}\right)$, then interface active power exchange levels are zero $p_{b^{T}, t}^{T}=0$ and if bus $b^{T}$ does not contain load, then $L_{b^{T}, t}^{T, p}=0$. The nodal power flow balance constraints for reactive power are similarly defined:

$$
\sum_{i \in \mathcal{I}_{b^{T}}^{T}} g_{i, t}^{T, q}+\sum_{\substack{l=1: \\ r(l)=b^{T}}}^{L} f_{l, t}^{T, q}+q_{b^{T}, t}^{T}=L_{b^{T}, t}^{T, q}+\sum_{\substack{l=1: \\ s(l)=b^{T}}}^{L} f_{l, t}^{T, q} .
$$

AC Power Flow Constraints in Rectangular Coordinates. Following [38]-[42], AC power flow is modeled in rectangular coordinates by using complex voltages $v_{b^{T}, t}^{T}=v_{b^{T}, t}^{T, R e}+j \cdot v_{b^{T}, t}^{T, I m}$.

In the complex plane, complex voltages can be represented as row vectors $v_{b^{T}, t}^{T}=\left(v_{b^{T}, t}^{T, R e}, v_{b^{T}, t}^{T, I m}\right)$ and power flows can be written as ${ }^{2}$

$$
\begin{aligned}
& f_{l, t}^{T, p}=v_{s(l), t}^{T} \cdot\left(\begin{array}{ll}
g_{s(l), r(l)} & -b_{s(l), r(l)} \\
b_{s(l), r(l)} & g_{s(l), r(l)}
\end{array}\right) \cdot\left(v_{r(l), t}^{T}\right)^{T}, \\
& f_{l, t}^{T, q}=v_{s(l), t}^{T} \cdot\left(\begin{array}{rr}
-b_{s}(l), r(l) & -g_{s(l), r(l)} \\
g_{s(l), r(l)} & -b_{s(l), r(l)}
\end{array}\right) \cdot\left(v_{r(l), t}^{T}\right)^{T} .
\end{aligned}
$$

Here $b_{s(l), r(l)}$ is susceptance and $g_{s(l), r(l)}$ is conductance of line $(s(l), r(l))$. Node $s(l)$ denotes the "sending" node of line $l$, and $r(l)$ denotes the "receiving" node of the line $l$.

Voltage Restrictions. The complex voltage within each node $b^{T}$ is subject to the following restrictions:

$$
\begin{aligned}
& \frac{v_{b^{T}}^{T} \leq \sqrt{\left(v_{b^{T}, t}^{T}\right) \cdot\left(v_{b^{T}, t}^{T}\right)^{T}}}{\sqrt{\left(v_{b^{T}, t}^{T}\right) \cdot\left(v_{b^{T}, t}^{T}\right)^{T}} \leq \overline{v_{b^{T}}^{T}}} .
\end{aligned}
$$

Transmission Capacity Constraints. Power flows in each line $l$ satisfy the following transmission capacity constraints:

$$
\sqrt{\left(f_{l, t}^{T, p}\right)^{2}+\left(f_{l, t}^{T, q}\right)^{2}} \leq \bar{f}_{l} \text {. }
$$

\section{B. DSO Model}

Consider a distribution network with a radial topology operated by a distribution system operator (DSO) and connected to a TSO's root bus $j$. Within each DSO, let $\mathcal{B}^{D}$ be a set of buses indexed by $b^{D}, \mathcal{I}_{b^{D}}^{D}$ be a set of generators at bus $b^{D}$ indexed by $i, \mathcal{L}^{D}$ be a set of transmission lines indexed by $l$. Objective. The DSO aims to minimize the total generation cost and to maximize the profit from selling power to the TSO. Therefore, the following sum of the two objectives is minimized as:

$$
\min _{g_{j}^{D}, p_{j}^{D}, q_{j}^{D}}\left\{O_{j}^{D}\left(g_{j}^{D}, p_{j}^{D}, q_{j}^{D} ; \boldsymbol{\Lambda}_{j}\right)\right\}=
$$

\footnotetext{
${ }^{2}$ Here superscript "T" indicates voltage at the TSO level, while " $\mathrm{T}$ " outside of the parentheses indicates the "transpose."
}

$$
\begin{aligned}
\min _{g_{j}^{D}, p_{j}^{D}, q_{j}^{D}} & \left\{\sum_{i \in \mathcal{I}_{b_{D}^{T}}^{D}, t \in \mathcal{T}}\left(C_{i, j, t}^{D, p} \cdot g_{i, j, t}^{D, p}+C_{i, j, t}^{D, q} \cdot g_{i, j, t}^{D, q}\right)\right. \\
& \left.-\sum_{t \in \mathcal{T}}\left(\lambda_{j, t}^{p} \cdot p_{j, t}^{D}+\lambda_{j, t}^{q} \cdot q_{j, t}^{D}\right)\right\}, j \in \mathcal{J}^{T},
\end{aligned}
$$

where $\boldsymbol{\Lambda}_{j}=\left\{\lambda_{j, t}^{p}, \lambda_{j, t}^{q}\right\}$ denote the locational marginal prices at the transmission bus $j$, which are a set of Lagrangian multipliers corresponding to nodal power flow balance constraints (9) and (10). Multipliers are the wholesale LMPs at the transmission root bus $j \in \mathcal{J}^{T}$ with an interconnected distribution system $j$. Within the distribution network, each conventional generator $i$ at hour $t$ produces active power denoted by $g_{i, j, t}^{D, p}$ and reactive power denoted by $g_{i, j, t}^{D, q}$. The corresponding active and reactive power generations costs are $C_{i, j, t}^{D, p}$ and $C_{i, j, t}^{D, q}$. Accordingly, the first summation within 16 accounts for the active and reactive power generation cost of conventional generators located within the distribution system $j$. The second summation within (16) accounts for the cost of transactions performed by the DSO in the wholesale electricity market in response to price signals $\lambda_{j, t}^{p}$ and $\lambda_{j, t}^{q}$. Capacity bids by the DSO in the wholesale market (referred in this paper as "interface power exchange amounts") are $p_{j, t}^{D}$ and $q_{j, t}^{D}$, which are positive if power exchange flow is from DSO to TSO, i.e., DSO sells power, and negative, otherwise.

Generation Capacity Constraints. Active $g_{i, j, t}^{D, p}$ and reactive $g_{i, j, t}^{D, q}$ generation levels of unit $i$ are constrained as:

$$
\underline{G}_{i, j}^{D, p} \leq g_{i, j, t}^{D, p} \leq \bar{G}_{i, j}^{D, p}, \underline{G}_{i, j}^{D, q} \leq g_{i, j, t}^{D, q} \leq \bar{G}_{i, j}^{D, q},
$$

where the minimum and maximum active power limits are $\underline{G}_{i, j}^{D, p}$ and $\bar{G}_{i, j}^{D, p}$, while the minimum and maximum reactive power limits are $\underline{G}_{i, j}^{D, q}$ and $\bar{G}_{i, j}^{D, q}$.

Second-Order Cone Relaxation of AC Power Flow. Since distribution systems are assumed to have a radial topology, AC power flows are modeled by using an exact second-order cone (SOC) relaxation following [17], [18]. The following constraint captures relationships among active $\left(f_{l, j, t}^{D, p}\right)^{2}$ and reactive $\left(f_{l, j, t}^{D, q}\right)^{2}$ power flows squared as well as current squared $a_{l, j, t}$ in line $l$, and voltage squared $v_{s(l), j, t}$ at the sending end $s(l)$ of line $l$ as:

$$
\left(f_{l, j, t}^{D, p}\right)^{2}+\left(f_{l, j, t}^{D, q}\right)^{2} \leq v_{s(l), j, t} \cdot a_{l, j, t} .
$$

To capture voltage drops across line $l$ between voltages squared at sending $s(l)$ and receiving $r(l)$ buses, the following constraint is used:

$$
\begin{aligned}
& v_{r(l), j, t}-v_{s(l), j, t}= \\
& 2 \cdot\left(R_{l, j} \cdot f_{l, j, t}^{D, p}+X_{l, j} \cdot f_{l, j, t}^{D, q}\right)-a_{l, j, t} \cdot\left(R_{l, j}^{2}+X_{l, j}^{2}\right),
\end{aligned}
$$

where parameters $R_{l, j}$ and $X_{l, j}$ are the reactanace and impedance of line $l$.

Since power flow at sending and receiving buses of each line $l$ differs due to losses incurred by transmission, the apparent power flow limit $\bar{S}_{l, j}$ is enforced for the sending and receiving buses separately within the following two sets of constraints:

$$
\begin{gathered}
\left(f_{l, j, t}^{p}\right)^{2}+\left(f_{l, j, t}^{q}\right)^{2} \leq\left(\bar{S}_{l, j}\right)^{2} \\
\left(f_{l, j, t}^{D, p}-a_{l, j, t} R_{l, j}\right)^{2}+\left(f_{l, j, t}^{D, q}-a_{l, j, t} X_{l, j}\right)^{2} \leq\left(\bar{S}_{l, j}\right)^{2} .
\end{gathered}
$$

Voltage Restriction Constraints. The bus voltages squared 
are limited by $\underline{v_{b^{D}, j}^{D}}$ and $\overline{v_{b^{D}, j}^{D}}$ as:

$$
\underline{v_{b^{D}, j}^{D}} \leq v_{b^{D}, j, t} \leq \overline{v_{b^{D}, j}^{D}} .
$$

Nodal Power Flow Balance Constraints. With the newly added load $L_{b^{D}, j, t}^{D, p}$, generation levels $g_{i, j, t}^{D, p}$ and interface power exchange amount $p_{j, t}^{D}$, the nodal power balance is enforced following [18, eq. (3)] as:

$$
\begin{aligned}
& \sum_{l \mid s(l)=b^{D}} f_{l, j, t}^{D, p}-\left(f_{l, j, t}^{D, p}-a_{l, j, t} \cdot R_{l, j}\right)_{l \mid r(l)=b^{D}}- \\
& \sum_{i \in \mathcal{I}_{b^{D}}^{D}} g_{i, j, t}^{D, p}+L_{b^{D}, j, t}^{D, p}+p_{j, t}^{D}+v_{b^{D}, j, t} \cdot G_{l \mid s(l)=b^{D}, j}=0
\end{aligned}
$$

where $G_{l, j}$ is conductance of line $l$. If bus $b^{D}$ does not contain a generator, then $\sum_{i \in \mathcal{I}_{b D}^{D}} g_{i, j, t}^{D, p}=0$, if bus $b^{D}$ is not a root bus $\left(b^{D} \notin \mathcal{J}^{T}\right)$, then interface active power exchange levels are zero $p_{j, t}^{D}=0$, and if bus $b^{D}$ does not contain load, then $L_{b^{D}, j, t}^{D, p}=0$. Because of the radial topology, the summation of power flows is performed with respect to lines whereby $b^{T}$ is a "sending" bus because any node can have several children nodes and at most one parent node. Thus, any node is able to receive power from only one parent, although with a loss of $a_{l, j, t} \cdot G_{l, j}$.

Nodal power flow for reactive power is similarly introduced:

$$
\begin{aligned}
& \sum_{l \mid s(l)=b^{D}} f_{l, j, t}^{D, q}-\left(f_{l, j, t}^{D, q}-a_{l, j, t} \cdot X_{l, j}\right)_{l \mid r(l)=b^{D}}- \\
& \sum_{i \in \mathcal{I}_{b^{D}}^{D}} g_{i, j, t}^{D, q}+L_{b^{D}, j, t}^{D, q}+q_{j, t}^{D}+v_{b^{D}, j, t} \cdot B_{l \mid s(l)=b^{D}}=0,
\end{aligned}
$$

where $B_{l, j}$ is susceptance of line $l$.

\section{Coordinated TSO-DSO Model}

Operating decisions of the TSO and multiple DSOs are coordinated by solving the following optimization problem:

$$
\begin{aligned}
& \min _{\substack{\mathbf{G}_{T}^{T}, \mathbf{P}^{T}, \mathbf{Q}^{T} \\
g_{j}^{D}, p_{j}^{D}, q_{j}^{D}}}\left\{O^{T}\left(\mathbf{G}^{\mathbf{T}}, \mathbf{P}^{\mathbf{T}}, \mathbf{Q}^{\mathbf{T}}\right)+\sum_{j \in \mathcal{J}^{T}} O_{j}^{D}\left(g_{j}^{D}, p_{j}^{D}, q_{j}^{D}, \mathbf{\Lambda}_{j}\right)\right\}, \\
& \text { s.t., (3) - 15, 17 }-124 .
\end{aligned}
$$

Moreover, the following interface power flow constraints ensure that the amount bought at the TSO level equals to the power sold at the DSO level, and vice versa.

\section{Interface Power Flow Constraints.}

$$
\mathbf{P}^{D}=\mathbf{P}^{T}, \mathbf{Q}^{D}=\mathbf{Q}^{T}
$$

where $\mathbf{P}^{D}, \mathbf{P}^{T}, \mathbf{Q}^{D}$, and $\mathbf{Q}^{T}$ are vectors that consist of $\left\{p_{j}^{D}\right\}$, $\left\{p_{j}^{T}\right\},\left\{q_{j}^{D}\right\},\left\{q_{j}^{T}\right\}$, respectively.

Moreover, as pointed out by [9], levels of flexibility provided by DSOs is impacted by feasible ranges of power, and $P Q$ interdependence needs to be captured at the TSO-DSO interface. Therefore, the following interface power exchange limit constraints are also imposed:

$$
\sqrt{\left(p_{j, t}^{T}\right)^{2}+\left(q_{j, t}^{T}\right)^{2}} \leq \overline{P Q}_{j}^{T}, \sqrt{\left(p_{j, t}^{D}\right)^{2}+\left(q_{j, t}^{D}\right)^{2}} \leq \overline{P Q}_{j}^{D} .
$$

The problem (25)-(28) is mixed-integer nonlinear programming (MINLP) problem, which is difficult in view of the combinatorial complexity introduced by the binary unit commitment variables within generation capacity (3)-(4) and ramprate constraints (5)-(8), and the non-linearity introduced by AC power flow (11)-(12), voltage restrictions (13)-(14), transmission capacity constraints (15) and the interface power flow restrictions (28). Moreover, cross-product terms within AC power flows (11)-(12) and voltage restrictions $(13)$ contribute to the non-convexity of the problem.

\section{Solution Methodology}

To resolve the above difficulties, the Surrogate AbsoluteValue Lagrangian Relaxation method [36] is used in a novel way to decompose problem (25)-27) into TSO and DSO subproblems after relaxing interface power flow constraints 27). To obtain location marginal prices $\boldsymbol{\Lambda}_{j}$, power flow balance constraints (9) and (10) are relaxed as explained in subsection IV.A. To overcome non-linearity difficulties at the TSO level, which arise because of cross-products within AC power flows (11) and (12), voltage restrictions (13)-(14), transmission capacity constraints 15 and interface power exchange limit constraints (28), novel dynamic linearization is then developed as explained in subsection IV.B. The entire algorithm is presented in subsection IV.C.

\section{A. Surrogate Absolute-Value Lagrangian Relaxation}

Relaxed Problem. After relaxing nodal flow balance (9)(10) and interface power exchange constraints (27), and after penalizing their violations, the relaxed problem becomes:

$$
\begin{aligned}
& \min _{\substack{\mathbf{G}^{T}, \mathbf{P}^{T}, \mathbf{Q}^{T} \\
g_{j}^{D}, p_{j}^{D}, q_{j}^{D}}} L_{c}\left(\mathbf{G}^{T}, \mathbf{P}^{T}, \mathbf{Q}^{T}, g_{j}^{D}, p_{j}^{D}, q_{j}^{D} ; \mathbf{\Lambda}\right)= \\
& \min _{\substack{\mathbf{G}^{T}, \mathbf{P}^{T}, \mathbf{Q}^{T} \\
g_{j}^{D}, p_{j}^{D}, q_{j}^{D}}}\left\{O^{T}\left(\mathbf{G}^{T}, \mathbf{P}^{T}, \mathbf{Q}^{T}\right)+\sum_{j \in \mathcal{J}^{T}} O_{j}^{D}\left(g_{j}^{D}, p_{j}^{D}, q_{j}^{D} ; \mathbf{\Lambda}_{j}\right)\right. \\
& \left.+\Lambda \cdot \mathbf{R}+c \cdot\|\mathbf{R}\|_{1}\right\} \text {, s.t., (3) - 8, (11) - 15, , 17) - 24 }
\end{aligned}
$$

where $\boldsymbol{\Lambda}_{j}=\left(\boldsymbol{\Lambda}_{j}^{P}, \boldsymbol{\Lambda}_{j}^{Q}\right)$ with $\boldsymbol{\Lambda}_{j}^{P}$ denoting multipliers that relax active power flow balance constraints $(9)$ and $\boldsymbol{\Lambda}_{j}^{Q}$ denoting multipliers that relax reactive power flow balance constraints (10). The vector $\boldsymbol{\Lambda}=\left(\boldsymbol{\Lambda}^{P}, \boldsymbol{\Lambda}^{Q}, \boldsymbol{\Psi}^{P}, \boldsymbol{\Psi}^{Q}\right)$ denotes a vector of Lagrangian multipliers, $\mathbf{R}=\left(\mathbf{B}^{P}, \mathbf{B}^{Q}, \mathbf{P}^{D}-\mathbf{P}^{T}, \mathbf{Q}^{D}-\mathbf{Q}^{T}\right)^{T}$ denotes a vector of constraint violations: $\mathbf{B}^{P}$ and $\mathbf{B}^{Q}$ denote violation levels of power flow balance constraints 9 - $-(10)$, and $\mathbf{P}^{D}-\mathbf{P}^{T}$ and $\mathbf{Q}^{D}-\mathbf{Q}^{T}$ are violation levels of interface power exchange constraints (27). The relaxed problem (29) is separated into TSO and DSO subproblems as explained next. TSO “Absolute-Value" Relaxed Subproblem.

$$
\begin{aligned}
& \min _{\mathbf{G}^{T}, \mathbf{P}^{T}, \mathbf{Q}^{T}}\left\{L_{c}^{T}\left(\mathbf{G}^{T}, \mathbf{P}^{T}, \mathbf{Q}^{T}\right)\right\}= \\
& \min _{\mathbf{G}^{T}, \mathbf{P}^{T}, \mathbf{Q}^{T}}\left\{O^{T}\left(\mathbf{G}^{T}, \mathbf{P}^{T}, \mathbf{Q}^{T}\right)+\boldsymbol{\Lambda} \cdot \mathbf{R}^{k-1}+c \cdot\left\|\mathbf{R}^{k-1}\right\|_{1}\right\}, \\
& \text { s.t., (3) - (8), (11) - 15), }
\end{aligned}
$$

where $\mathbf{R}^{k-1}=\left(\mathbf{B}^{P}, \mathbf{B}^{Q}, \mathbf{P}^{D, k-1}-\mathbf{P}^{T}, \mathbf{Q}^{D, k-1}-\mathbf{Q}^{T}\right)^{T}$ is defined in a similar way to $\mathbf{R}$ above, except that interface power exchange values from the DSO $\mathbf{P}^{D, k-1}$ and $\mathbf{Q}^{D, k-1}$ are not decision variables, rather, they are fixed values obtained at the previous iteration $k-1$. After solving $(30)$, values $\mathbf{G}^{T, k}$, $\mathbf{P}^{T, k}, \mathbf{Q}^{T, k}, \mathbf{B}^{P, k}$ and $\mathbf{B}^{Q, k}$ are obtained, and $\mathbf{P}^{T, k}$ and $\mathbf{Q}^{T, k}$ are passed on to the DSO subproblem discussed next. 
DSO “Absolute-Value” Relaxed Subproblem.

$$
\begin{gathered}
\min _{g_{j}^{D}, p_{j}^{D}, q_{j}^{D}}\left\{O_{j}^{D}\left(g_{j}^{D}, p_{j}^{D}, q_{j}^{D} ; \mathbf{\Lambda}_{j}\right)+\right. \\
\left(\mathbf{\Psi}^{P}, \mathbf{\Psi}^{Q}\right) \cdot\left(\mathbf{P}^{D}-\mathbf{P}^{T, k}, \mathbf{Q}^{D}-\mathbf{Q}^{T, k}\right)^{T}+ \\
\left.c \cdot\left\|\left(\mathbf{P}^{D}-\mathbf{P}^{T, k}, \mathbf{Q}^{D}-\mathbf{Q}^{T, k}\right)\right\|_{1}\right\}, \\
\text { s.t., 17 - 24). }
\end{gathered}
$$

The DSOs' decisions to buy/sell power from the TSO is affected not only by the nodal shadow prices $\boldsymbol{\Lambda}_{j}$ but also by multipliers $\left(\Psi^{P}, \Psi^{Q}\right)$ - the shadow prices that correspond to interface power exchange constraints (27). After solving DSO subproblems [31, values $\mathbf{G}^{D, k} \equiv\left\{g_{j}^{D, k}\right\}, \mathbf{P}^{D, k} \equiv\left\{p_{j}^{D, k}\right\}$ and $\mathbf{Q}^{D, k} \equiv\left\{q_{j}^{D, k}\right\}$ are obtained, and $\mathbf{P}^{D, k}$ and $\mathbf{Q}^{D, k}$ are passed on to the TSO subproblem to be used in the next iteration.

Multiplier Update. To coordinate the TSO and DSO subproblems above, multipliers are updated as follows:

$$
\mathbf{\Lambda}^{k}=\mathbf{\Lambda}^{k-1}+s^{k} \cdot \mathbf{R}^{k},
$$

where $\mathbf{R}^{k}=\left(\mathbf{B}^{P, k}, \mathbf{B}^{Q, k}, \mathbf{P}^{D, k}-\mathbf{P}^{T, k}, \mathbf{Q}^{D, k}-\mathbf{Q}^{T, k}\right)^{T}$. Values $\mathbf{P}^{T, k}, \mathbf{Q}^{T, k}$ as well as constraint violations $\mathbf{B}^{P, k}$ and $\mathbf{B}^{Q, k}$ are obtained by solving the TSO subproblem 30 and $\mathbf{P}^{D, k}, \mathbf{Q}^{D, k}$ are obtained by solving DSO subproblems (31). Stepsize Update. Following [36], the stepsize is updated in the following way:

$$
s^{k}=\alpha^{k-1} \cdot s^{k-1} \cdot \frac{\left\|\mathbf{R}^{k-1}\right\|_{2}}{\left\|\mathbf{R}^{k}\right\|_{2}} .
$$

where $\alpha^{k-1}$ is a step-sizing parameter

$$
\alpha^{k-1}=1-\frac{1}{M \cdot k^{1-\frac{1}{k^{r}}}}, M>1, r>0 .
$$

Penalty Coefficient Update. In the beginning of the iterative process, the penalty coefficient $c^{k}$ increases by a predetermined constant $\beta>1$ :

$$
c^{k}=c^{k-1} \cdot \beta .
$$

The intent is to increase the value of $c^{k}$ until the norm of constraint violations reduces to zero and a feasible solution is obtained, after which the penalty coefficient is decreased per

$$
c^{k}=c^{k-1} \cdot \beta^{-1}
$$

Subsequently, the penalty coefficient is not increased.

\section{B. Practical Considerations of the Method}

In practical implementation, the following considerations are important. 1. Voltage restrictions (13)-(14), transmission capacity constraints (15) and interface power exchange limits (28) are nonlinear, they need to be appropriately linearized while maintaining convergence and feasibility. 2. Because of binary commitment decision variables, the TSO problem is combinatorial and further decomposition is needed. These considerations are discussed next.

\section{Non-linearity of AC Power Flow and Voltage Restriction Constraints at TSO.}

1. Linearization of Cross-Product Terms. AC power flow constraints contain cross-products of voltages at neighboring buses. To linearize these constraints, voltages at one of the buses $(s(l)$ or $r(l))$ are fixed, thereby rendering the constraint linear. Assuming that voltages at "sending" buses are fixed at values $v_{s(l), t}^{T, k-1}$ obtained at previous iteration $k-1$, the constraint becomes:

$$
f_{l, t}^{T, p}=v_{s(l), t}^{T, k-1} \cdot\left(\begin{array}{rr}
g_{s(l), r(l)} & -b_{s(l), r(l)} \\
b_{s(l), r(l)} & g_{s(l), r(l)}
\end{array}\right) \cdot\left(v_{r(l), t}^{T}\right)^{T} .
$$

The issue with this approach is that a subset of "receiving" buses does not generally equal to the entire set of buses. As a result, some of the voltages are not updated. To overcome this issue, voltages at "sending" buses are fixed, and voltages at "receiving" buses are fixed independently based on (11) and the average of the resulting power flows is taken as:

$$
\begin{aligned}
& f_{l, t}^{T, p}=\frac{1}{2} v_{s(l), t}^{T, k-1} \cdot\left(\begin{array}{cc}
g_{s(l), r(l)} & -b_{s(l), r(l)} \\
b_{s(l), r(l)} & g_{s(l), r(l)}
\end{array}\right) \cdot\left(v_{r(l), t}^{T}\right)^{T}+ \\
& \frac{1}{2} v_{s(l), t}^{T} \cdot\left(\begin{array}{ll}
g_{s(l), r(l)} & -b_{s(l), r(l)} \\
b_{s(l), r(l)} & g_{s(l), r(l)}
\end{array}\right) \cdot\left(v_{r(l), t}^{T, k-1}\right)^{T} .
\end{aligned}
$$

In doing so, all voltages are updated at every iteration. Reactive power flows are linearized in the same way.

In the following, linearization of voltage restrictions (14), transmission capacity constraints $(15)$ and interface power flow restrictions 28) is performed. Since voltage restrictions (13) delineate non-convex set, their lineaization is treated separately afterward.

2. Linearization of (14), (15) and (28). Since the above constraints are similar in their structure, the lineaization is demonstrated by using constraints (14). The linearization is performed in two steps. In Step 1, equation (14) is squared to eliminate the non-linearity introduced by the square root and thus can equivalently be rewritten as:

$$
\left(v_{b^{T}, t}^{T, R e}\right)^{2}+\left(v_{b^{T}, t}^{T, I m}\right)^{2} \leq\left(\bar{v}_{b^{T}}^{T}\right)^{2}
$$

In Step 2, squared terms within (39) are then linearized in the following way:

$$
v_{b^{T}, t}^{T, R e, k-1} \cdot v_{b^{T}, t}^{T, R e}+v_{b^{T}, t}^{T, I m, k-1} \cdot v_{b^{T}, t}^{T, I m} \leq\left(\bar{v}_{b^{T}}^{T}\right)^{2} .
$$

Linearization of (15) and 28) is performed by using exactly the same procedure.

3. Linearization of (13). The first two steps of linearization follow those described above. Additionally, to avoid possible infeasibility, the following "soft" constraint with the penalty variable $v_{b^{T}, t}^{T, \text { pen }}$ is enforced following [45] as:

$$
\left(\underline{v}_{b^{T}}^{T}\right)^{2}-v_{b^{T}, t}^{T, p e n} \leq v_{b^{T}, t}^{T, R e, k-1} \cdot v_{b^{T}, t}^{T, R e}+v_{b^{T}, t}^{T, I m, k-1} \cdot v_{b^{T}, t}^{T, I m} .
$$

To enforce feasibility of 41], $v_{b^{T}, t}^{T, p e n}$ are penalized. To avoid getting trapped at a local minimum, which may happen when penalties are high, a novel "flexible penalization" is introduced by using Lagrangian multipliers in a non-conventional way without relaxing (41). The Lagrangian multipliers increase only when the "soft" constraints are violated $\left(v_{b^{T}, t}^{T, p e n}>0\right)$, and decrease when satisfied: $\left(v_{b^{T}, t}^{T, p e n}=0\right)$ and $\left(\underline{v}_{b^{T}}^{T}\right)^{2}<$ $v_{b^{T}, t}^{T, R e, k-1} \cdot v_{b^{T}, t}^{T, R e}+v_{b^{T}, t}^{T, I m, k-1} \cdot v_{b^{T}, t}^{T, I m}$. When constraints are satisfied, the associated multipliers become zero. To discourage future violations of these constraints, violations $v_{b^{T}, t}^{T, p e n}$ are also penalized by using $c^{k}$ as will be shown below.

To ensure that solutions satisfying constraints 38, (40) and (41) satisfy the original constraints (13)-(14), (15) and 28), proximal-like terms $\left\|\mathbf{V}^{T}-\mathbf{V}^{T, k-1}\right\|_{1}$, which capture the deviations of voltages from previously obtained values are first 
introduced and then penalized by $c^{k}$. Here $\mathbf{V}^{T}$ is a vector of voltages $\left(\left\{v_{b^{T}, t}^{T, R e}\right\},\left\{v_{b^{T}, t}^{T, I m}\right\}\right)$. Following the same logic, to satisfy (15) and (28), the following terms are introduced $\left\|\mathbf{F}^{T}-\mathbf{F}^{T, k-1}\right\|_{1}$ and $\left\|\mathbf{P Q}^{T}-\mathbf{P Q}^{T, k-1}\right\|_{1}$, where $\mathbf{F}^{T}$ is a vector of AC power flows $\left(\left\{f_{l, t}^{T, p}\right\},\left\{f_{l, t}^{T, q}\right\}\right)$ and $\mathbf{P Q}^{T}$ is a vector of interface power exchange flows $\left(\left\{p_{j, t}^{T}\right\},\left\{q_{j, t}^{T}\right\}\right)$.

Linearized TSO Subproblem. The resulting TSO subproblem then becomes:

$$
\begin{gathered}
\min _{\mathbf{G}^{T}, \mathbf{P}^{T}, \mathbf{Q}^{T}}\left\{L_{c}^{T}\left(\mathbf{G}^{T}, \mathbf{P}^{T}, \mathbf{Q}^{T}\right)+c^{k} \cdot\left\|\mathbf{V}^{T}-\mathbf{V}^{T, k-1}\right\|_{1}+\right. \\
c^{k} \cdot\left\|\mathbf{F}^{T}-\mathbf{F}^{T, k-1}\right\|_{1}+c^{k} \cdot\left\|\mathbf{P} \mathbf{Q}^{T}-\mathbf{P Q}^{T, k-1}\right\|_{1}+ \\
\left.c^{k} \cdot \sum_{t \in \mathcal{T}, b^{T} \in \mathcal{B}^{T}} v_{b^{T}, t}^{T, p e n}+\sum_{t \in \mathcal{T}, b^{T} \in \mathcal{B}^{T}} \lambda_{b^{T}, t}^{T, p e n} \cdot v_{b^{T}, t}^{T, p e n}\right\},
\end{gathered}
$$

$$
\text { s.t., (3) - 8, (11) - 15. }
$$

Flexibility and versatility of Lagrangian multipliers not only allows to coordinate subsystems in the inter-subproblem way but also to resolve non-convexity in the intra-subproblem manner to avoid local minima which may be caused by (13). The multiplier update for (42) is operationalized by appending constraints violations $\mathbf{R}$ by $v_{b^{T}, t}^{T, p e n}$ and multipliers $\Lambda$ by $\lambda_{b^{T}, t}^{T, p e n}$, and by following the multiplier updating procedure described in 32 with projections of negative values of $\lambda_{b^{T}, t}^{T, p e n}$ onto a subspace $\{\lambda \mid \lambda \geq 0\}$. Piece-wise linear $l_{1}$-norms within (42) are linearized following standard procedures [35], [36].

Combinatorial Complexity. While traditional decomposition cannot be used because of cross-products of voltages, subproblems with reduced complexity can still be formed by fixing commitment statuses of selected units (e.g., statuses of $75 \%$ of all units) at values $x_{i, t}^{k-1}$ obtained at previous iterations. After fixing variables in such a way, the resulting TSO subproblem (42) is much easier to solve.

Algorithm. The algorithm is summarized below:

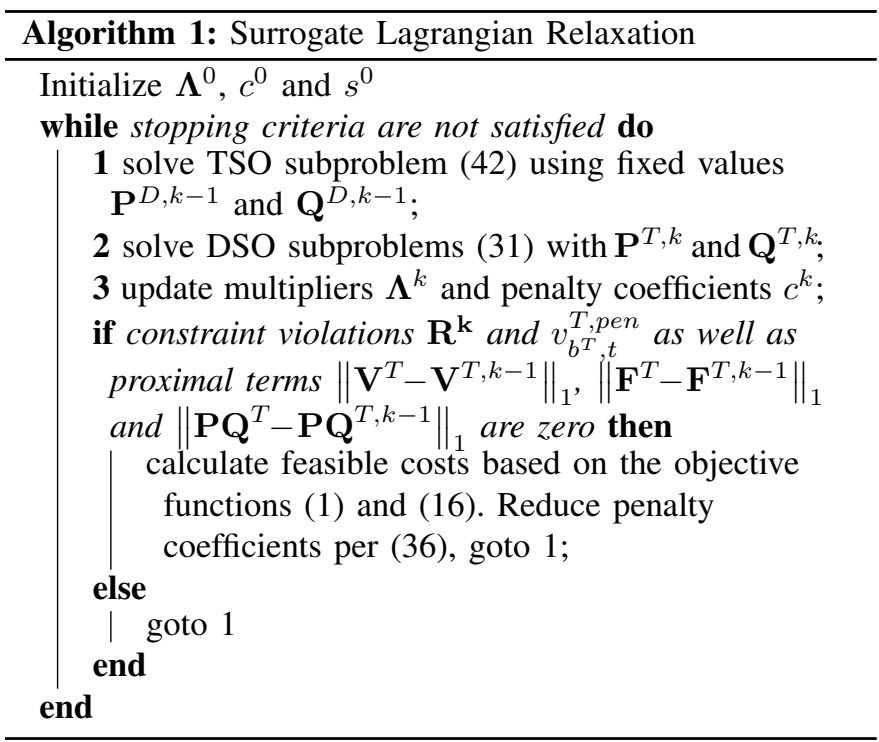

Stopping Criteria. The algorithm is terminated after the stopping criteria are satisfied. The following stopping criteria may be used: CPU time limit, number of iterations, surrogate subgradient norm, distance between multipliers, etc.

\section{NumericAl Testing}

The TSO-DSO coordination approach developed in this paper is implemented in CPLEX 12.10 .0 .0 by using a laptop with the processor Intel@ Xeon ${ }^{\circledR}$ CPU E3-1535M v6 @ 3.1$\mathrm{GHz}$ and $32.00 \mathrm{~GB}$ of RAM. Three case studies are considered to illustrate the important concepts: convergence of multipliers, the satisfaction of nonlinear constraints, scalability, and cost benefits for TSO and DSOs. Within Case Study 1, a small 1-hour example with 4 buses 2 generators within TSO, and one DSO is considered to demonstrate the convergence of multipliers. Within Case Study 2, a small 9-bus system [43] is considered to demonstrate the efficiency of linearization and convergence of voltages to satisfy constraints (13)-(14). Within Case Study 3, 4-, 8- and 16-hour instances with 1 TSO modeled using IEEE 118-bus system [43] and 32 DSOs modeled using IEEE 34-bus systems [43] are considered to demonstrate the methods ability to coordinate a large number of DSO and the cost advantage of the coordination.

\section{A. Case Study 1: Simple TSO-DSO Coordination Example.}

Consider a simple TSO-DSO topology with the corresponding technical characteristics such as resistance and inductance as well as generation costs, power interface exchange prices and loads (Fig. 11): TSO consists of 4 buses, includes 2 generators (located at buses 1 and 4) and containing one root bus (bus 2) whereby one DSO is connected modeled using data for the IEEE 34-bus system. The resulting dispatch including power generation and power flow as well as values of multipliers at convergence are also shown in Fig. 1 below.

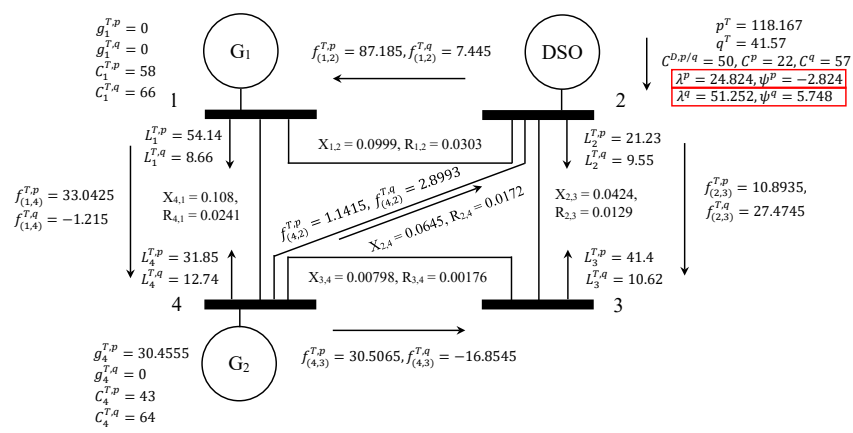

Fig. 1. Topology of the TSO-DSO system for Case Study 1.

As demonstrated in Fig. 1 to serve the loads, TSO purchases power from the $\mathrm{DSO} p_{2}^{T}=118.167, q_{2}^{T}=41.57$ and generates power using generator at bus $4: g_{4}^{T, p}=30.4555$.

Fig. 2 below demonstrates convergence of the multipliers at the root bus.

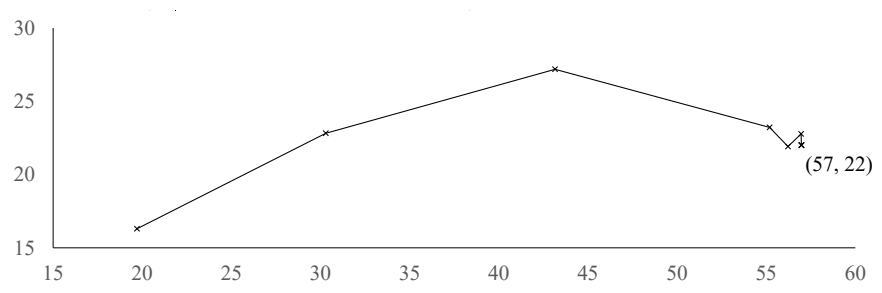

Fig. 2. Convergence of $\lambda_{2}^{p}+\psi_{2}^{p}$ and $\lambda_{2}^{q}+\psi_{2}^{q}$ at the root bus 2 . 
Multipliers $\lambda_{2}^{p}$ and $\lambda_{2}^{q}$ are shadow nodal prices, and $\psi_{2}^{p}$ and $\psi_{2}^{q}$ are shadow prices associated with interface power exchange constraints. Total shadow prices associated with the TSO-DSO power exchange are $\lambda_{2}^{p}+\psi_{2}^{p}$ and $\lambda_{2}^{q}+\psi_{2}^{q}$ as shown in Fig. 2. Prices approach the values of $\$ 22$ for active and $\$ 57$ for reactive power, which are exactly equal to the DSO's interface power exchange amount bids $C_{2}^{p}$ and $C_{2}^{q}$.

To demonstrate the cost-advantage of TSO-DSO coordination, a series of 30 Monte Carlo simulations is performed by randomly generating generation costs, as well as DSO's bids, by using a uniform distribution U[20,60]. On average, DSOs' total cost is $4.18 \%$ lower and TSO's cost is $13.57 \%$ lower as compared to DSOs and TSO cost obtained by solving DSO and TSO problems separately.

\section{B. Case Study 2: Illustration of Efficiency of Dynamic Lin- earization Based on the 9-bus System.}

Within this example, consider a 9-bus system [43]. The purpose of this example is to demonstrate that the dynamic linearization is efficient and that at convergence solutions satisfy original constraints. For illustration purposes, the satisfaction with respect to voltage restriction constraints (13)-(14) is demonstrated. The results are shown in Fig. 3 below.

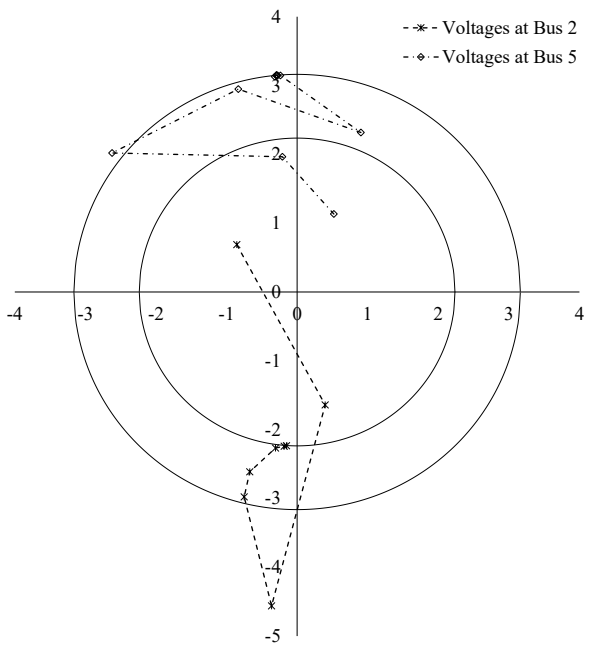

Fig. 3. Convergence of voltages for Example 2 for selected buses. Rea voltages are along the $\mathrm{x}$-axis and imaginary voltages are along the $\mathrm{y}$-axis.

As shown in Fig. 3, the inner and outer circles are the boundaries of the feasible region corresponding to minimal and maximum voltages squared $\left(v_{b^{T}}^{T}\right)^{2}$ and $\left(\overline{v_{b^{T}}^{T}}\right)^{2}$, respectively. The feasible region is in-between the two circles. Within buses 2 and 5, initial voltages are within the inner circle and are infeasible. Through coordination and penalization of constraint violations, voltages converge to values within the feasible region, i.e., original constraints 13 - 14 are satisfied.

\section{Case Study 3: TSO-DSO Coordination with One IEEE 118-} Bus System, 32 IEEE 34-Bus Systems

Consider a TSO-DSO system with TSO modeled as IEEE 118-bus system, and DSOs modeled as IEEE 34-bus systems. For the cost comparison purposes, the "uncoordinated" TSO problem is solved by itself using the method presented in Section IV by updating penalty coefficients per (35)- 36 and by updating multipliers per (32) without including $\mathbf{P}^{D, k}-\mathbf{P}^{T, k}$ and $\mathbf{Q}^{D, k}-\mathbf{Q}^{T, k}$ into $\mathbf{R}^{k}$. The DSO problems are directly solved by using CPLEX, which can handle the types of nonlinearities that are present within the DSO problems. The total costs obtained are then compared with the cost obtained by the TSO-DSO coordination and the results are summarized in Table I. Relative cost improvement is shown in parentheses.

TABLE I

COST IMPROVEMENT THROUGH TSO-DSO COORDINATION FOR 1 TSO AND 32 DSOS WITH 4-, 8- AND 12-HOUR HORIZONS.

\begin{tabular}{cccccc}
\hline \hline $\begin{array}{c}\text { No. } \\
\text { of hrs. }\end{array}$ & $\begin{array}{c}\text { TSO cost } \\
(\times \$ 1000)\end{array}$ & $\begin{array}{c}\text { DSO cost } \\
(\times \$ 1000)\end{array}$ & $\begin{array}{c}\text { Total cost } \\
(\times \$ 1000)\end{array}$ & $\begin{array}{c}\text { TSO } \\
\text { time }(\mathrm{s})\end{array}$ & $\begin{array}{c}\text { DSO } \\
\text { time }(\mathrm{s})\end{array}$ \\
\hline 4 & 337.65 & 41.88 & 379.54 & 305.79 & 113.26 \\
& $(\downarrow 27.53 \%)$ & $(\downarrow 21.88 \%)$ & $(\downarrow 26.95 \%)$ & & \\
8 & 653.39 & 84.65 & 738.04 & 717.83 & 289.52 \\
& $(\downarrow 26.87 \%)$ & $(\downarrow 21.01 \%)$ & $(\downarrow 26.24 \%)$ & & \\
12 & 1113.46 & 87.76 & 1201.23 & 1335.38 & 486.15 \\
& $(\downarrow 22.68 \%)$ & $(\downarrow 45.40 \%)$ & $(\downarrow 24.96 \%)$ & & \\
\hline
\end{tabular}

As demonstrated in Table I, the method is capable of coordinating a large number of DSOs together with the TSO, and that the both systems benefit from the coordination.

\section{CONCLUSION}

This paper presents a novel formulation for coordination of TSO and multiple DSOs by considering both active and reactive power through the inclusion of nonlinear AC power flow constraints within both systems, and by considering binary UC decisions. The resulting combinatorial and the highly nonlinear problem is solved by using the decomposition and coordination Surrogate "Absolute-Value" Lagrangian Relaxation together with the novel dynamic linearization to satisfy nonlinear constraints. Case studies demonstrate the effectiveness of coordination through the convergence of multipliers, efficiency of linearization, and that the method is capable of coordinating a large number of DSOs, and that both the transmission and distribution systems benefit from the proposed coordination. The new solution methodology opens several directions for 1) handling non-linear constraints that delineate convex as well as non-convex feasible regions, specifically, to handle AC power flow within unit commitment problems, 2) inclusion of stochastic elements to efficiently handle uncertainties while solving unit commitment problem with meshed topology with AC flow without losses of accuracy due to simplifications frequently used, and 3) game-theoretic studies to investigate strategies TSO and DSOs behaviors.

\section{REFERENCES}

[1] Z. Yuan and M. R. Hesamzadeh, "Hierarchical Coordination of TSO-DSO Economic Dispatch Considering Large-Scale Integration of Distributed Energy Resources," Applied Energy, vol. 195, pp. 600-615, Jun. 2017.

[2] I. Pérez-Arriaga and A. Bharatkumar, "A Framework for Redesigning Distribution Network Use of System Charges Under High Penetration of Distributed Energy Resources: New Principles for New Problems,' Tech. rep., CEEPR Working Paper Series no. WP-2014-006; 2014.

[3] A. Papalexopoulos, R. Frowd, and A. Birbas, "On the Development of Organized Nodal Local Energy Markets and a Framework for the TSODSO Coordination," Electric Power Systems Research, vol. 189, 2020. 
[4] G. Migliavacca, M. Rossi, D. Six, M. Dzamarija, S. Horsmanheimo, C. Madina, I. Kockar, and J.M. Morales, "SmartNet: A H2020 Project Analysing TSO-DSO Interaction to Enable Ancillary Services Provision from Distribution Networks," CIRED, Glasgow, Scotland, June 2017.

[5] G. Migliavacca, M. Rossi, H. Gerard, M. Dzamarija, S. Horsmanheimo, C. Madina, I. Kockar, G. Leclecq, M. Marroquín, H. Svendsen, "TSODSO Coordination and Market Architectures for an Integrated Ancillary Services Acquisition: the View of the SmartNet Project," CIRED, Paris, France, June 2018.

[6] M. Rossi, G. Viganò, G. Migliavacca, Y. Vardanyan, R. Ebrahimi, G. Leclercq, P. Sels, M. Pavesi, T. Gueuning, J. Jimeno, N. Ruiz, G. Howorth, J. Camargo, C. Hermans, F. Spiessen, and H. Svendsen, "Testing TSODSO Interaction Schemes for the Participation of Distribution Energy Resources in the Balancing Market: the SmartNet Simulator," CIRED, Madrid, Spain, June 2019.

[7] EDSO. "General Guidelines for Improving TSO-DSO Cooperation." Tech. rep.; 2015.

[8] EDSO. "TSO-DSO Data Management Report." Tech. rep.; 2015.

[9] J. P. Silva, J. Sumaili, R. J. Bessa, L. Seca, M. Matos, and V. Miranda, "The Challenges of Estimating the Impact of Distributed Energy Resources Flexibility on the TSO/DSO Boundary Node Operating Points," Computers and Operations Research, vol. 96, pp. 294-304, 2018.

[10] J. P. Silva, J. Sumaili, R. J. Bessa, L. Seca, M. Matos, V. Miranda, M. Caujolle, B. Goncer-Maraver, and M. Sebastian-Viana, "Estimating the Active and Reactive Power Flexibility Area at the TSO-DSO Interface," IEEE Transactions of Power Systems, vol. 33, no. 5, pp. 4741-4750, Sep. 2018.

[11] F. Capitanescu, "TSO-DSO Interaction: Active Distribution Network Power Chart for TSO Ancillary Services Provision," Electric Power Systems Research, vol. 163, part A, pp. 226-230, 2018.

[12] M. Zipf and D. Möst, "Cooperation of TSO and DSO to Provide Ancillary Services," 2016 13th International conference on the European energy market (EEM), pp. 1-6, 2016

[13] I. Gómez et al., "Cost-Benefit Analysis of the Selected National Cases," SmartNet Project, Tech. Rep. D4.3, 2019

[14] M. Rossi, G. Migliavacca, G. Viganò, D. Siface, C., I. Gomez, I. Kockar, and Andrei Morch, "TSO-DSO Coordination to Acquire Services from Distribution Grids: Simulations, Cost-benefit Analysis and Regulatory Conclusions from the SmartNet Project," Electric Power Systems Research, vol. 189, 2020.

[15] H. Jain, K. Rahimi, A. Tbaileh, R. P. Broadwater, A. K. Jain and M. Dilek, "Integrated Transmission \& Distribution System Modeling and Analysis: Need \& Advantages," In proceedings of the IEEE Power and Energy Society 2016 General Meeting, Boston, MA, July 2016.

[16] A. Hassan and Y. Dvorkin, "Energy Storage Siting and Sizing in Coordinated Distribution and Transmission Systems," IEEE Transactions on Sustainable Energy, vol. 9, no. 4, pp. 1692-1701, Oct. 2018.

[17] S. S. Torbaghan, G. Suryanarayana, H. Höschle, R. D'hulst, F. Geth, C. Caerts, and D. Van Hertem, "Optimal Flexibility Dispatch Problem Using Second-Order Cone Relaxation of AC Power Flows," IEEE Transactions on Power Systems, vol. 35, No. 1, pp. 98-108, 2020.

[18] M. Farivar and S. H. Low, "Branch Flow Model: Relaxations and Convexification - Part I," IEEE Transactions on Power Systems, vol. 28, no. 3, pp. 2554-2564, Aug. 2013.

[19] E. Polymeneas and S. Meliopoulos, "Aggregate Modeling of Distribution Systems for Multi-Period OPF," 2016 Power Systems Computation Conference (PSCC), Genoa, 2016, pp. 1-8, doi: 10.1109/PSCC.2016.7540987.

[20] Z. Li, Q. Guo, H. Sun, and J. Wang, "Coordinated Transmission and Distribution AC Optimal Power Flow," IEEE Transactions on Smart Grid, vol. 9, no. 2, pp. 1228-1240, Jun 2016.

[21] Z. Yuan, M.R. Hesamzadeh, and D.R. Biggar, "Distribution Locational Marginal Pricing by Convexified ACOPF and Hierarchical Dispatch," IEEE Transactions on Smart Grid, vol. 9, no. 4, pp. 3133-3142, 2018.

[22] A. Saint-Pierre, and P. Mancarella, "Active Distribution System Management: A Dual Horizon Scheduling Framework for DSO/TSO Interface Under Uncertainty," IEEE Transactions on Smart Grid, vol. 8, no. 5 , 2186-2197, 2017.

[23] M. Rossi, et al., "Testing TSO-DSO Interaction Schemes for the Participation of Distributed Energy Resources in the Balancing Market: the Smartnet Simulator," Presented at the 25th International Conference on Electricity Distribution, Madrid, Spain, 2019.

[24] H. Le Cadre, I. Mezghani, A. Papavasiliou, "A Game-Theoretic Analysis of Transmission-Distribution System Operator Coordination," European Journal of Operational Research, vol. 274, no. 1, pp. 317-339, 2019.

[25] A. Mohammadi, M. Mehrtash, A. Kargarian, "Diagonal Quadratic Approximation for Decentralized Collaborative TSO+DSO Optimal Power
Flow," IEEE Transactions on Smart Grid, vol. 10, no. 3, pp. 2358-2370, 2019.

[26] A. Papavasiliou, and I. Mezghani, "Coordination Schemes for the Integration of Transmission and Distribution System Operations," Presented at the Power Systems Computation Conference (PSCC), Dublin, Ireland, 2018.

[27] Z. Li, Q. Guo, H. Sun, and J. Wang, "Coordinated Economic Dispatch of Coupled Transmission and Distribution Systems Using Heterogeneous Decomposition," IEEE Transactions on Power Systems, vol. 31, no. 6, pp. 4817-4830, Nov. 2016

[28] Z. Li, Q. Guo, H. Sun, and J. Wang, "A New LMP-SensitivityBased Heterogeneous Decomposition for Transmission and Distribution Coordinated Economic Dispatch" IEEE Transactions on Smart Grid, vol. 9, no. 2, pp. 931-941, May 2016.

[29] M. Caramanis, E. Ntakou, W. W. Hogan, A. Chakrabortty and J. Schoene, "Co-Optimization of Power and Reserves in Dynamic T\&D Power Markets With Nondispatchable Renewable Generation and Distributed Energy Resources," Proceedings of the IEEE, vol. 104, no. 4, pp. 807-836, April 2016.

[30] A. Givisiez, K. Petrou, and L. Ochoa, "A Review on TSO-DSO Coordination Models and Solution Techniques," Electric Power Systems Research, vol. 189, 2020 . doi:10.1016/j.epsr.2020.106659

[31] M. A. Bragin, and Y. Dvorkin, “ Toward Coordinated Transmission and Distribution Operations," In proceedings of the IEEE Power and Energy Society 2018 General Meeting, Portland, Oregon

[32] A. Kargarian, and Y. Fu, "System of Systems Based SecurityConstrained Unit Commitment Incorporating Active Distribution Grids," IEEE Transactions of Power Systems, vol. 29, no. 5, pp. 2489-2498, Sep. 2014.

[33] A. Nawaz, H. Wang, Q. Wu, and M. Kumar Ochani, "TSO and DSO with Large-Scale Distributed Energy Resources: A Security Constrained Unit Commitment Coordinated Solution," International Transactions on Electrical Energy Systems, vol. 30, no. 3, 2020. https://doi.org/10.1002/20507038.12233

[34] M. A. Bragin, P. B. Luh, J. H. Yan, N. Yu, and G. A. Stern "Convergence of the Surrogate Lagrangian Relaxation Method," Journal of Optimization Theory and Applications, vol. 164, no. 1, 2015, pp. 173-201, DOI: 10.1007/s10957-014-0561-3.

[35] X. Sun, P. B. Luh, M. A. Bragin, Y. Chen, J. Wan, and F. Wang, "A Decomposition and Coordination Approach for Large-Scale Security Constrained Unit Commitment Problems with Combined Cycle Units," IEEE Transactions on Power Systems, vol. 33, no. 5, pp. 5297-5308, Sept. 2018. DOI: 10.1109/PESGM.2017.8274098

[36] M. A. Bragin, P. B. Luh, B. Yan, and X. Sun. "A Scalable Solution Methodology for Mixed-Integer Linear Programming Problems Arising in Automation," IEEE Transactions of Automation Science and Engineering, vol. 16, no. 2, pp. 531 - 541, Jun. 2018.

[37] D. Gurevin, S. Zhou, L. Pepin, B. Li, M. A. Bragin, C. Ding, and F. Miao, "A Surrogate Lagrangian Relaxation-Based Model Compression for Deep Neural Networks." https://arxiv.org/pdf/2012.10079.pdf

[38] G. L. Torres, V. H. Quintana, and G. Lambert-Torres, "Optimal Power Flow on Rectangular Form Via an Interior Point Method," in IEEE North American Power Symposium, 1996.

[39] V. da Costa, N. Martins, and J. L. R. Pereira, "Developments in the Newton Raphson Power Flow Formulation Based on Current Injections," IEEE Transactions on Power Systems, vol. 14, no. 4, pp. 1320-1326, November 1999.

[40] X. P. Zhang, S. G. Petoussis, and K. R. Godfrey, "Nonlinear Interior Point Optimal Power Flow Method Based on a Current Mismatch Formulation," IEEE Proceedings-Generation, Transmission and Distribution, vol. 152, no. 6, pp. 795-805, November 2005.

[41] X. Bai, H.Wei, K. Fujisawa, and Y.Wang, "Semidefinite Programming for Optimal Power Flow problems," International Journal of Electrical Power and Energy Systems, vol. 30, no. 6-7, pp. 383-392, 2008.

[42] Q. Li , L. Yang and S. Lin, "Coordination Strategy for Decentralized Reactive Power Optimization Based on a Probing Mechanism,“ IEEE Transactions on Power Systems, vol. 30, no. 2, pp. 555-562, Mar. 2015.

[43] R. D. Zimmerman, C. E. Murillo-Sanchez (2020). MATPOWER (Version 7.1) [Software]. Available: https://matpower.org. Accessed: 01/23/21

[44] B. Yan, P. B. Luh, E. Litvinov, T. Zheng, D. Schiro, M. A. Bragin, F. Zhao, J. Zhao, and I. Lelic, "A Systematic Formulation Tightening Approach for Unit Commitment Problems," IEEE Transactions on Power Systems, vol. 35, no. 1, pp. 782-794, Aug. 2019.

[45] N. Raghunathan, M. A. Bragin, B. Yan, P. B. Luh, K. Moslehi, X. Feng, Y. Yu, C.-N. Yu, and C.-C. Tsai, "Exploiting Soft Constraints within Decomposition and Coordination Methods for Sub-Hourly Unit Commitment," TechRxiv. Preprint. https://doi.org/10.36227/techrxiv.12950414.v1 